\title{
Laparoscopic sleeve gastrectomy for morbidly obese adolescents in Singapore
}

\author{
Dallan Dargan ${ }^{1}$, MBвch, MSc, Dmitrii Dolgunov ${ }^{1}$, MRCs, Khin Thida Soe ${ }^{2}$, MBBs, Pamela $\underline{E r}^{3}$, BSc, Dip Dietetics, \\ Fathimath $\underline{\text { Naseer }}^{3}$, BSc, Davide Lomanto ${ }^{1,2}$, MD, PhD, Jimmy BY $\underline{\text { So }}^{1,2}$, FRCS, MPH, Asim Shabbir ${ }^{1,2}$, MBBS, MMed
}

INTRODUCTION Laparoscopic sleeve gastrectomy (LSG) outcomes among adolescents and factors associated with adolescent obesity in Singapore were evaluated.

METHODS Prospectively collected data of patients aged 16-19 years who underwent LSG was retrospectively reviewed. A lifestyle questionnaire, Berlin and Epworth scores, and Patient Health Questionnaire-9 scores were collected. Preoperative anthropometrics, comorbidities, weight loss and body composition outcomes were recorded.

RESULTS Among 208 LSGs, 13 (6.3\%) were performed on obese adolescents. Mean age and body mass index (BMI) at first presentation were $19.1 \pm 0.9$ (range 16.8-19.8) years and $46.2 \pm 6.3$ (range 36-57) kg/m², respectively. There was family history of obesity $(n=7)$ and regular consumption of high-calorie drinks $(n=12)$. Most patients had comorbidities $(n=12)$, including hypertension $(n=5)$, asthma $(n=4)$, diabetes mellitus $(n=3)$, hernia $(n=3)$ and obstructive sleep apnoea requiring continuous positive airway pressure support $(n=3)$. At one year, excess weight loss was $64.3 \% \pm 34.7 \%$ (range $21.8 \%-101.5 \%$ ), while BMI and fat mass dropped to $31.2 \pm 7.6$ (range $23-40$ ) $\mathrm{kg} / \mathrm{m}^{2}$ and $17.4 \mathrm{~kg}$, respectively. Pain score was $2 / 10$ at 24 hours after surgery. Mean postoperative stay was 2.7 days. No complications or readmissions occurred. Remission of diabetes mellitus and hypertension was reported in two of three and four of five adolescents, respectively, within one year of surgery.

CONCLUSION LSG is a safe option for adolescents with good short-term weight loss outcomes and remission of metabolic comorbid conditions.

Keywords: adolescent obesity, body mass index, excess weight loss, laparoscopic sleeve gastrectomy, metabolic surgery

\section{INTRODUCTION}

Worldwide, the number of obese individuals has doubled since 1980. ${ }^{(1)}$ Every year, approximately 3.4 million people die as a consequence of being overweight or obese, according to the World Health Organization. (1) The increased prevalence of adolescent obesity in affluent countries correlates with per capita income, and Singapore is no exception. Adolescent obesity is potentially one of the most serious health problems of the 21 st century. Childhood obesity tends to persist into adulthood and poses a risk of developing into metabolic conditions, such as diabetes mellitus, hypertension and cardiovascular disease, at a younger age. ${ }^{(2-7)}$ Obesity prevalence among persons in Singapore aged 18-69 years has increased from $6.9 \%$ in 2004 to $10.8 \%$ in $2010 .^{(8)}$ In adults aged $18-29$ years in Singapore, $15.4 \%$ of men and $5.8 \%$ of women were obese (body mass index [BMI] $\left.\geq 30 \mathrm{~kg} / \mathrm{m}^{2}\right)$ in 2010 . $^{(8)}$

Efforts at lifestyle modification and use of pharmacotherapy to improve the health of morbidly obese adolescents are often unsuccessful. As obese children and adolescents are predisposed to a range of obesity-related disorders during growth and have increased risk of adult obesity with associated cardiovascular risk, early treatment is therefore essential. ${ }^{(9,10)}$ More adolescents and their families are seeking surgical treatment for morbid obesity because of a lack of response to behavioural, diet and medical treatment approaches. ${ }^{(11)}$ Bariatric surgery is in demand worldwide and is gaining much support and acceptance in Asia. ${ }^{(12,13)}$ Among morbidly obese adolescents, bariatric surgery has been associated with resolution of concomitant metabolic conditions. ${ }^{(14,15)}$ The most commonly performed procedures are laparoscopic sleeve gastrectomy (LSG), laparoscopic gastric bypass and gastric banding. ${ }^{(16)}$ Among these, sleeve gastrectomy is increasingly being utilised for morbidly obese adults and adolescents, and has been shown to be more effective than gastric banding for adolescents. ${ }^{(17)}$ The premise for using sleeve gastrectomy rather than the latter two options includes the relative simplicity of the surgical procedure and lower risk of long-term complications. ${ }^{(18)}$

According to the American Society for Metabolic and Bariatric Surgeons (ASMBS), the number of adolescents who underwent bariatric surgery increased in the late 1990s. In 2005, among members of the ASMBS, 53\% had performed bariatric surgery on obese adolescents. In 2003, more than 100 hospitals performed bariatric surgical procedures on obese adolescents, with a mean age of 16 years. ${ }^{(19)}$ The Obesity Surgery Society of Australia and New Zealand has set criteria that adolescents aged 15 years and above with associated morbidity should be considered for obesity surgery. ${ }^{(11)}$

To our knowledge, there are only a handful of studies on bariatric surgery for morbidly obese Asian adolescents. ${ }^{(13,20-24)}$ Our study sought to provide an overview of adolescent bariatric surgery in a multiethnic population from Singapore.

${ }^{1}$ Department of Surgery, National University Hospital, ${ }^{2}$ Yong Loo Lin School of Medicine, National University of Singapore, ${ }^{3}$ Department of Dietetics, National University Hospital, Singapore

Correspondence: Dr Asim Shabbir, Senior Consultant, Department of Surgery, National University Hospital, NUHS Tower Block, Level 8, 1 E Kent Ridge Road, Singapore 119228. asim_shabbir@nuhs.edu.sg 


\section{METHODS}

Adolescents aged $\leq 19$ years who underwent bariatric surgery (i.e. LSG) at National University Hospital, Singapore, from November 2010 to July 2014 were selected. We performed a retrospective analysis of the data, which was collected prospectively and entered into our database, as approved by the institutional review board (IRB no. NUH-2012/00114). A questionnaire was completed for each patient at first presentation to the obesity management clinic. Data on dietary, exercise, cigarette and alcohol habits, family history of obesity, and the Berlin questionnaire, ${ }^{(25)}$ Epworth Sleepiness Scale scores ${ }^{(26)}$ and Patient Health Questionnaire-9 (PHQ-9) scores $^{(27)}$ was recorded by specialist nurses. Demographic, anthropometric and postoperative outcomes were retrieved from the database. Variables such as age, gender, BMI before and after surgery, length of hospital stay, complications, absolute weight loss, percentage excess weight loss, changes in body composition and remission of comorbidities were recorded. Blood pressure, fasting blood glucose (FBG), glycated haemoglobin (HbA1c) and serum lipids were checked at each visit. Adolescents with hypertension, diabetes mellitus or dyslipidaemia were assessed for remission according to ASMBS guidelines. ${ }^{(28)}$ The Tanita ${ }^{\circledR}$ body composition analyser (model TBF-300; Tanita Corporation, Tokyo, Japan) was used for all patients. Pain scores were recorded using the Visual Analogue Scale. Complications were defined as adverse events that required medical or surgical intervention after surgery.

Data analysis and visualisation was aided by Microsoft Excel version 2010 (Microsoft Corporation, Redmond, WA, USA), QI Macros ${ }^{\circledR}$ for Excel (KnowWare International Inc, Denver, CO, USA) and Daniel's XL Toolbox add-in for Excel version 6.60 (Daniel Kraus, Würzburg, Germany) software packages. Weightrelated calculations, including $\mathrm{BMI}$, were reported as mean \pm standard deviation. A one-tailed paired $t$-test was used to compare preoperative and one-year postoperative mean body composition measurements, changes in blood pressure and serum lipid indices. Statistical significance was set at $\mathrm{p}<0.05$.

\section{RESULTS}

The eight boys and five girls who underwent LSG $(n=13)$ had a mean age of $19.1 \pm 0.9$ (range 16.8-19.8) years at the first visit to the weight management clinic. This patient group constituted $6.3 \%$ of a total of 208 LSG procedures performed at our institution during the study period. Ethnically, the patients were Chinese $(\mathrm{n}=6,46.2 \%)$, Indian $(\mathrm{n}=4,30.8 \%)$ and Malay $(\mathrm{n}=3,23.1 \%)$. Table I lists the smoking, alcohol and dietary habits, and the prevalence of comorbidities among obese adolescents undergoing LSG. High-calorie drinks were frequently consumed. 7 (53.8\%) patients reported a family history of obesity. Surgery was offered to those who had tried lifestyle modification for weight loss but had failed to lose weight or maintain weight after loss, according to the ASMBS paediatric committee guidelines. ${ }^{(29)}$ Cigarette and alcohol use was infrequent among the group.

At presentation, $4(30.8 \%)$ patients had one comorbid condition while 8 (61.5\%) patients had two or more comorbidities. Hypertension, diabetes mellitus, asthma, hyperlipidaemia and hernia were observed (Table I). Irregular menses was noted in most
Table I. Behavioural and lifestyle variables associated with adolescent obesity $(n=13)$.

\begin{tabular}{lc}
\hline Variable & No. (\%) \\
\hline Smoking history & $1(7.7)$ \\
Smoker & $1(7.7)$ \\
Ex-smoker & $11(84.6)$ \\
Non-smoker & \\
History of alcohol consumption & $3(23.1)$ \\
Yes & $10(76.9)$ \\
No & \\
Diet & $12(92.3)$ \\
High-calorie drinks & $11(84.6)$ \\
Sweets & $9(69.2)$ \\
Snacks & $7(53.8)$ \\
Fried foods* & \\
Weight loss attempt by lifestyle modification* & $7(53.8)$ \\
Yes & $3(23.1)$ \\
No & \\
Family history of obesity* & $7(53.8)$ \\
Yes & $3(23.1)$ \\
No & \\
Comorbidity & $5(38.5)$ \\
Hypertension & $4(30.8)$ \\
Asthma & $3(23.1)$ \\
Hiabetes mellitus & $3(23.1)$ \\
Hyperlipidaemia & $2(15.4)$ \\
No. of comorbidities at presentation & $1(7.7)$ \\
I 2 & \\
\hline
\end{tabular}

*Three patients did not respond.

obese girls. In one patient, irregular menses was associated with hyperprolactinaemia and bilateral ovarian cystectomy. 3 (23.1\%) patients required continuous positive airway pressure support for sleep apnoea. All 13 patients scored positively in two or more categories on the Berlin questionnaire. In 3 (23.1\%) patients, this was associated with an Epworth score $>10$, indicating a high likelihood of sleep apnoea. $8(66.7 \%)$ of 12 patients had PHQ-9 scores $>4$ (mean 7.67, range 4-18), indicating at least mild depressive symptoms in most participants.

The mean length of postoperative hospital stay was 2.7 (range 2-4) days. All patients ambulated freely on the first postoperative day and drank clear fluids within 24 hours of surgery. There were no mortalities, complications or reoperations to report. The mean pain score at 24 hours after surgery, recorded for eight of 13 patients, was 2 out of 10 .

Of 13 patients, 5 (38.5\%) completed the one-year review, and $3(23.1 \%)$ completed three years of follow-up. At one year, the mean absolute weight was $39.3 \mathrm{~kg}$ lower than that before surgery (Fig. 1); mean body weight was $133.6 \pm 21.7$ (range 99.1-163.7) $\mathrm{kg}$ before surgery and $94.1 \pm 25.0$ (range 59.4-120.1) $\mathrm{kg}$ at one year after LSG $(p<0.01)$. The percentage excess weight loss for obese adolescents undergoing LSG at one year was $64.3 \%$ 
Table II. Changes in body composition of obese adolescents after surgery.

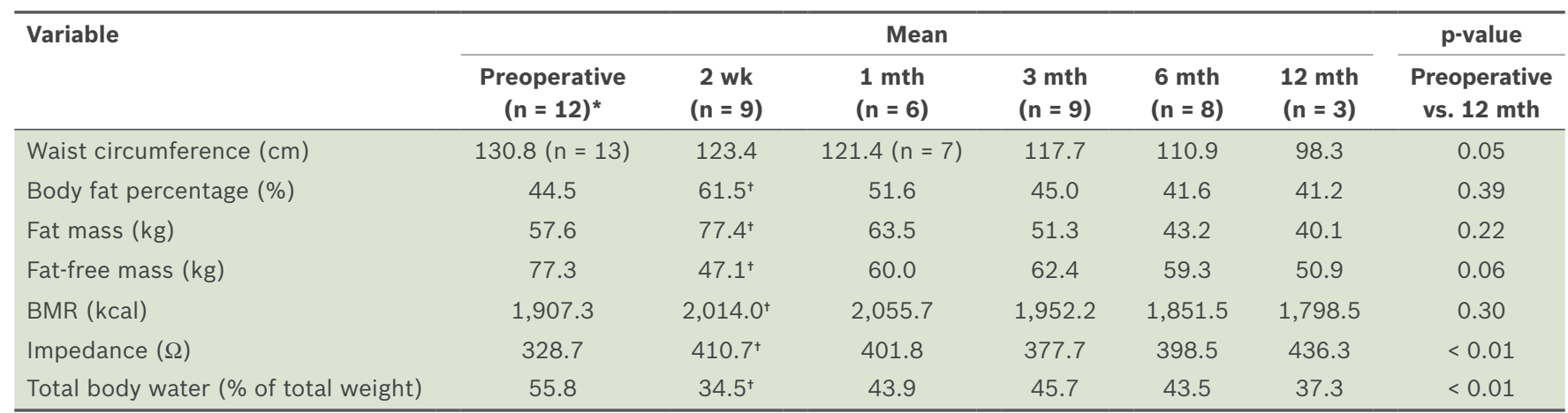

*One patient did not have preoperative values recorded for body fat, fat mass, fat-free mass and basal metabolic rate (BMR). †Recorded values for body fat percentage, fat mass and BMR were higher at two weeks than before surgery in most patients, while fat-free mass was lower. Contributing factors during this period were changes in bioelectrical impedance (due to changes in intracellular and extracellular water) and reduced total body water, rather than actual fat gain.

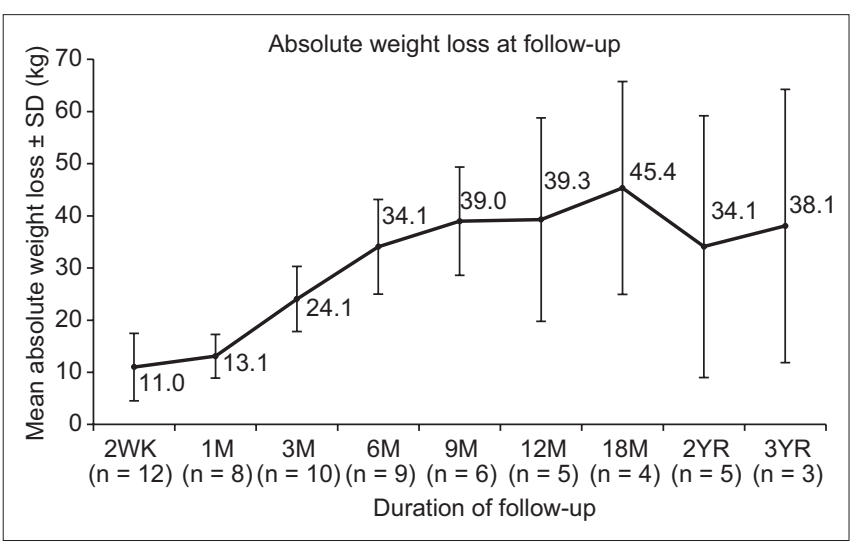

Fig. 1 Mean absolute weight loss of obese adolescents after surgery M: month; SD: standard deviation; WK: week; YR: year

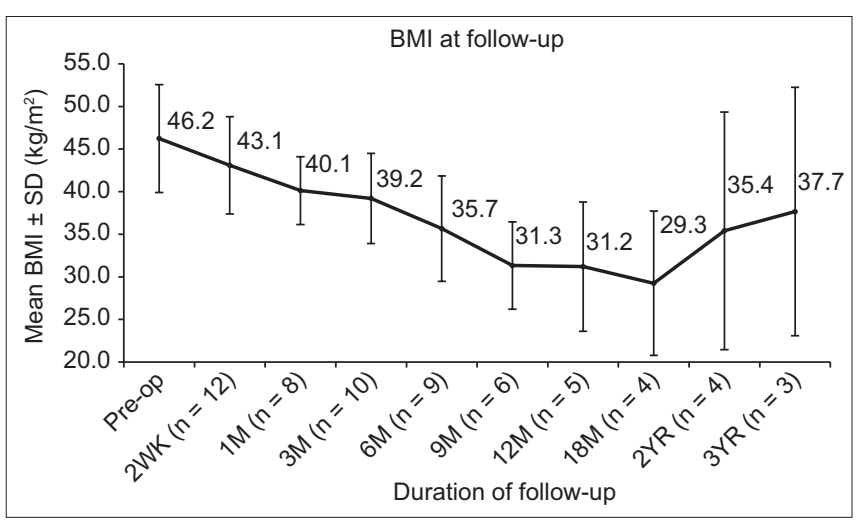

Fig. 3 Mean body mass index (BMI) of obese adolescents before and after surgery. M: month; preop: preoperative; SD: standard deviation; WK: week; YR: year

$\pm 34.7 \%$ (range $21.8 \%-101.5 \%$ ) (Fig. 2), while the mean BMI, which was $46.2 \pm 6.3$ (range $36-57$ ) $\mathrm{kg} / \mathrm{m}^{2}$ preoperatively, decreased to $31.2 \pm 7.6$ (range $23-40) \mathrm{kg} / \mathrm{m}^{2}(\mathrm{p}<0.0001)$ (Fig. 3).

Apart from weight loss, we found substantial changes in body composition one year after surgery. Table II shows a progressive reduction in mean body fat percentage, mean fat mass, waist circumference and excess weight loss. Energy consumption rate (i.e. basal metabolic rate) rose postoperatively, as did values for fat mass and body fat percentage, all of which were measured using bioelectrical impedance. This reflected alterations in the body's electrical

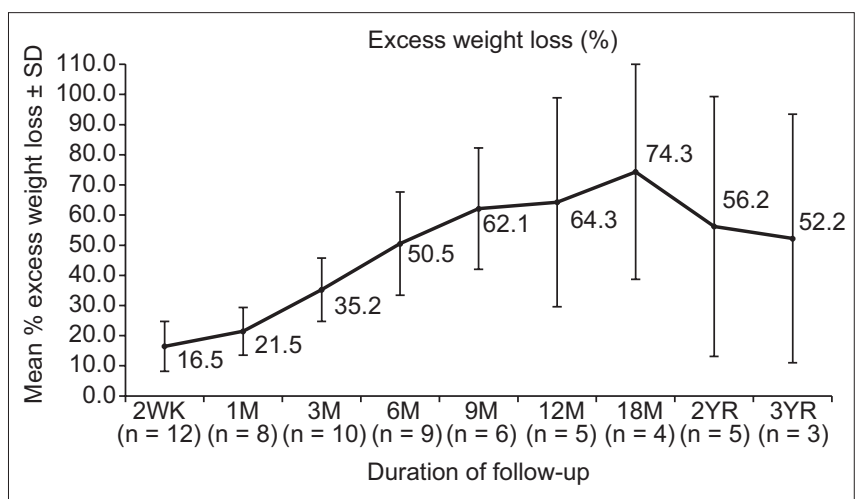

Fig. 2 Mean percentage excess weight loss of obese adolescents after surgery. M: month; SD: standard deviation; WK: week; YR: year

resistance in relation to fluctuations in body water during this period and response to surgery.

According to ASMBS outcome reporting standards, ${ }^{(28)}$ full remission of diabetes mellitus is declared if $\mathrm{FBG}<5.5 \mathrm{mmol} / \mathrm{L}$ and $\mathrm{HbA} 1 \mathrm{c}<6.5 \%$ in the absence of diabetic medications. Of the three diabetic adolescents who underwent LSG, two discontinued antidiabetic medications following surgery - one had complete remission at six months (FBG $4.3 \mathrm{mmol} / \mathrm{L}, \mathrm{HbA} 1 \mathrm{c} 5.3 \%$ ) and the other had improvement at three months (FBG $5.3 \mathrm{mmol} / \mathrm{L}, \mathrm{HbA} 1 \mathrm{c}$ $6.7 \%)$, with recurrence later.

With regard to hypertension before surgery, according to ASMBS guidelines, five patients had prehypertension (systolic blood pressure [SBP] 120-139 mmHg, diastolic blood pressure [DBP] 80-89 $\mathrm{mmHg}$ ), three had Stage 1 hypertension (SBP 140-159 mmHg, DBP 90-99 mmHg) and one had Stage 2 hypertension ( $\mathrm{SBP} \geq 160 \mathrm{mmHg}$, DBP $\geq 100 \mathrm{mmHg}$ ). Two patients proceeded to complete remission by one year (Table III, Patients 7 \& 8), five demonstrated improvement and one had no improvement (Table III, Patient 1). A further one patient (Patient 6) had complete remission when reviewed at two years. The mean SBP of these nine patients, which was $142.8 \mathrm{mmHg}$ preoperatively, fell to $131.5 \mathrm{mmHg}$ for those reviewed at one year $(p=0.06)$. One of two patients taking antihypertensive medications prior to surgery no longer required medication.

Fasting serum lipid evaluation demonstrated significant reductions in triglycerides $(p=0.01)$ and total cholesterol/ 
Table III. Systolic and diastolic blood pressure values of hypertensive or prehypertensive adolescents $(\mathrm{n}=9)$ before and after surgery.

\begin{tabular}{|c|c|c|c|c|c|c|c|c|c|c|}
\hline \multirow[t]{2}{*}{ Patient no. } & \multicolumn{5}{|c|}{ Systolic blood pressure $(\mathrm{mmHg})$} & \multicolumn{5}{|c|}{ Diastolic blood pressure $(\mathrm{mmHg})$} \\
\hline & Preoperative & $3 \mathrm{mth}$ & $6 \mathrm{mth}$ & $12 \mathrm{mth}$ & p-value & Preoperative & 3 mth & $6 \mathrm{mth}$ & $12 \mathrm{mth}$ & p-value \\
\hline 1 & 139 & 145 & 109 & 141 & & 86 & 96 & 66 & 85 & \\
\hline 2 & 155 & 145 & 137 & 140 & & 100 & 76 & 76 & 84 & \\
\hline 3 & 169 & 132 & - & 135 & & 84 & 70 & - & 85 & \\
\hline 4 & 145 & 153 & 140 & - & & 70 & 88 & 76 & - & \\
\hline 5 & 135 & 136 & 130 & - & & 82 & 89 & 71 & - & \\
\hline 6 & $155^{*}$ & - & - & - & & $92^{*}$ & - & - & - & \\
\hline 7 & 131 & 113 & 117 & - & & 83 & 61 & 69 & - & \\
\hline 8 & 128 & 103 & 106 & 110 & & 74 & 60 & 58 & 71 & \\
\hline 9 & 128 & 111 & 127 & - & & 70 & 69 & 75 & - & \\
\hline Mean & 142.8 & 129.8 & 123.7 & 131.5 & $0.06^{+}$ & 82.3 & 76.1 & 70.1 & 81.3 & $0.15^{+}$ \\
\hline
\end{tabular}

*Patient 6 attended review at two years from surgery and was normotensive (systolic blood pressure $120 \mathrm{mmHg}$, diastolic blood pressure $70 \mathrm{mmHg}$ ). + Based on one-tailed paired Student's $t$-test comparing preoperative and 12-month measurements.

Table IV. Fasting serum lipid analysis of obese adolescents before and after surgery.

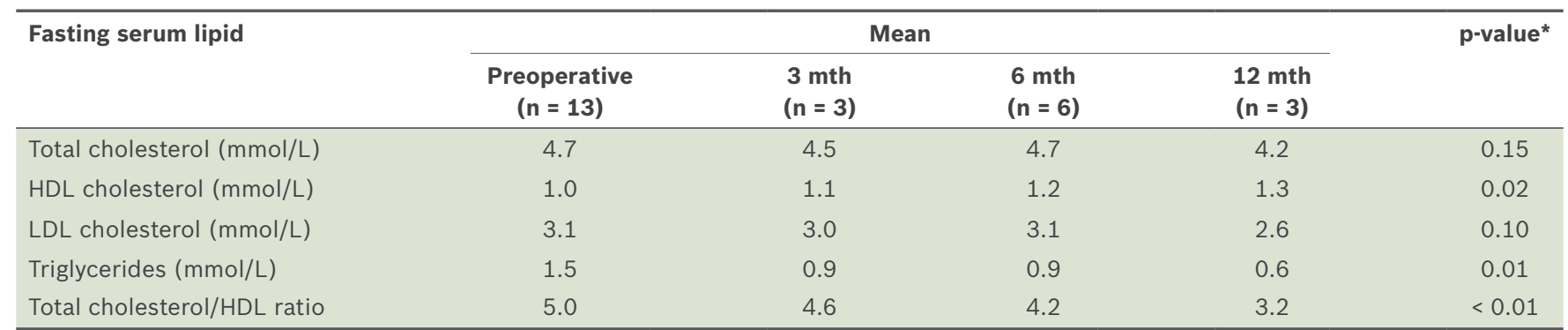

*Based on one-tailed paired Student's t-test comparing preoperative and 12-month measurements. HDL: high-density lipoprotein; LDL: low-density lipoprotein

Table V. ASMBS categories of dyslipidaemia among obese adolescents before and after surgery.

\begin{tabular}{|c|c|c|c|c|}
\hline \multirow[t]{2}{*}{ Variable } & \multicolumn{4}{|c|}{ No. (\%) } \\
\hline & Preoperative $(n=13)$ & 3 mth $(n=3)$ & 6 mth $(n=7)$ & 12 mth $(n=3)$ \\
\hline \multicolumn{5}{|l|}{ Total cholesterol (mmol/L)* } \\
\hline Normal $(<5.17)$ & $9(69.2)$ & $3(100.0)$ & $5(71.4)$ & $3(100.0)$ \\
\hline Borderline (range 5.17-6.20) & $3(23.1)$ & 0 & $2(28.6)$ & 0 \\
\hline High $(>6.20)$ & $1(7.7)$ & 0 & 0 & 0 \\
\hline \multicolumn{5}{|l|}{ Triglycerides (mmol/L) } \\
\hline Normal $(<1.69)$ & $9(69.2)$ & $3(100.0)$ & $7(100.0)$ & $3(100.0)$ \\
\hline Borderline high (range 1.69-2.25) & $1(7.7)$ & 0 & 0 & 0 \\
\hline High (range $2.26-5.65$ ) & $3(23.1)$ & 0 & 0 & 0 \\
\hline Very high (> 5.65) & 0 & 0 & 0 & 0 \\
\hline \multicolumn{5}{|l|}{ HDL (mmol/L) } \\
\hline Low $(<1.03)$ & $10(76.9)$ & $2(66.7)$ & $3(42.9)$ & $1(33.3)$ \\
\hline Normal (range 1.03-1.55) & $3(23.1)$ & 0 & $4(57.1)$ & $1(33.3)$ \\
\hline High (> 1.55) & 0 & $1(33.3)$ & 0 & $1(33.3)$ \\
\hline \multicolumn{5}{|l|}{ LDL (mmol/L) } \\
\hline Optimal $(<2.59)$ & $3(23.1)$ & 0 & $1(14.3)$ & $2(66.7)$ \\
\hline Near optimal (range 2.59-3.34) & $7(53.8)$ & $2(66.7)$ & $3(42.9)$ & $1(33.3)$ \\
\hline Borderline high (range 3.35-4.13) & $2(15.4)$ & $1(33.3)$ & $3(42.9)$ & $0(0)$ \\
\hline High (range 4.14-4.90) & $1(7.7)$ & 0 & 0 & 0 \\
\hline Very high $(>4.90)$ & 0 & 0 & 0 & 0 \\
\hline \multicolumn{5}{|l|}{ Total cholesterol/HDL ratio (mmol/L) } \\
\hline$<1 / 2$ average risk $(<3.27)$ & 0 & $1(33.3)$ & $2(28.6)$ & $2(66.7)$ \\
\hline $1 / 2$ to average risk (range $3.27-4.44$ ) & $6(46.2)$ & 0 & $3(42.9)$ & $1(33.3)$ \\
\hline $1-2 \times$ average risk (range $4.45-7.05$ ) & $6(46.2)$ & $2(66.7)$ & $2(28.6)$ & 0 \\
\hline $2-3 \times$ average risk (range $7.06-11.04$ ) & $1(7.7)$ & 0 & 0 & 0 \\
\hline
\end{tabular}

*Units were converted from mg/dL to mmol/L, as per American Society for Metabolic and Bariatric Surgeons (ASMBS) guidelines. HDL: high-density lipoprotein; LDL: low-density lipoprotein 
high-density lipoprotein $(\mathrm{HDL})$ ratio $(\mathrm{p}<0.01)$, and a significant increase in HDL cholesterol $(p=0.02)$ at one year (Table IV). The categories of dyslipidaemia, listed according to review period, are shown in Table V. Of two patients receiving lipid-lowering agents prior to surgery, one had an optimal lipid profile with values within the normal range at three months, indicating remission, while the other patient continued to require lipid-lowering medication at the three-year review.

\section{DISCUSSION}

Our study sought to determine the outcomes of LSG in an obese adolescent population from Singapore. The study's many strengths included its prospectively collected data, definitive follow-up schedules and treatment protocols. The finding of metabolic improvement highlights the clinical significance of LSG for treating not only obesity but also inducing remission of associated comorbidities, which is arguably more important. Long-term outcomes of sleeve gastrectomy in adults indicate that, on average, more than $60 \%$ excess BMI loss is maintained at over ten years from surgery, even as de novo gastro-oesophageal reflux occurred in $8 \%$ of patients in Europe. ${ }^{(30)}$ Similar findings have been reported at five years in Asia. ${ }^{(31)}$ These recent findings, published after the completion of our study, attest to the durability of the procedure in adults. While our cohort showed excellent excess and absolute weight loss, and improvement in BMI at 12 and 18 months, the results at three years were not as good.

After LSG, effective weight loss was achieved in morbidly obese adolescents in our study, with effective resolution of comorbidities. We suggest early postoperative weight-bearing exercise to prevent muscle loss in this group. The initial apparent rise in fat mass, body fat percentage and basal metabolic rate represents a change in the body's electrical resistance, as measurements were made using bioelectrical impedance. Impedance calculations are based on total body water, and intracellular and extracellular water values. ${ }^{(32)}$ Changes in the water-electrolyte balance of tissues, along with acute changes in body mass, are limitations of the use of bioelectrical impedance. ${ }^{(32)}$ A reduction in total body water was noted in each participant at the two-week review.

A decrease in fat-free mass was also noted in our series. Fat-free mass constitutes not only muscle mass but total body water, bone density and minerals. ${ }^{(15)}$ Supplementation of vitamins and minerals is compulsory to minimise the risk of nutritional deficiency. ${ }^{(15,33)}$ Although the values for fat-free mass at two weeks may relate to total body water, protein catabolism in the first month may be more rapid than fat catabolism. By one year, the values for reduction in fat mass and fat-free mass converge. This reflects the nutritional status of bariatric patients after surgery and suggests a need for better support infrastructure and more early advice to prevent muscle loss. Regular follow-up after surgery is compulsory for active weight management, ${ }^{(11)}$ and we also advocate resistance exercise to preserve muscle mass. Fat-free mass helps to dispose of excess nutrients, such as glucose, and is preserved by having adequate intake of proteins and regular exercise as part of an active lifestyle.
Ensuring the safety of LSG is a concern for adolescent patients, family and healthcare providers. Like other studies, the cases in our series had an absence of complications and rapid recovery enabling early discharge from hospital. Results from a series of 108 paediatric and adolescent patients from King Saud University Hospitals in Riyadh, Saudi Arabia, demonstrated LSG to be a safe and effective procedure. ${ }^{(21)}$ A series of 22 patients from Korea reported the high rate of resolution of comorbidities. ${ }^{(20)}$ Our institution keeps abreast of the outcomes of local and international adolescent bariatric surgery and constantly studies the risks and benefits of these procedures. Long-term results in this category of treatment, such as this study and efforts at other institutions, will explore the sustainability of bariatric surgery. ${ }^{(34)}$ For obese adolescents, the effectiveness of bariatric surgery is well established. Among the different surgical options, LSG has become more popular in recent years due to good outcomes and its perceived simplicity when compared with other bariatric procedures such as laparoscopic biliopancreatic diversion with duodenal switch and Roux-en-Y gastric bypass. ${ }^{(35)}$ Additionally, LSG may be followed by gastric bypass for further weight loss, if required. ${ }^{(36)}$ Possible long-term nutritional risks, sustainability of weight loss and resolution of comorbid conditions should be adequately evaluated. ${ }^{(3)}$ Questions still exist about how families can best support such lifestyle changes.

Psychopathologies are common in obese adolescents, and depressive disorder symptoms were observed among $66.7 \%$ of our cohort, which is at the higher end of the $15 \%-70 \%$ range noted in the literature. ${ }^{(37)}$ Meanwhile, the reported ranges for anxiety disorder and eating disorder symptoms are $15 \%-33 \%$ and $48 \%-70 \%$, respectively, prior to surgery, although these symptoms improve considerably after surgery. ${ }^{(37)}$ If lifestyle modifications fail after surgery, those adolescents would gain weight despite the metabolic modification effects of surgery. The durability and efficacy of this surgery in young adults are partly dependent on the motivation of patients to adhere to the postoperative dietary and lifestyle regimens that are necessary to maintain the success of the weight loss procedure.

This study was not without limitations. Although we only had 13 patients, with over $50 \%$ being lost to follow-up at one year and further attrition noted at three years, the study provided preliminary data for early outcomes of LSG that may be valuable for future studies. The rate of loss to follow-up may reflect a satisfactory outcome in a majority of cases, adding a negative bias to the results, and efforts to counteract attrition should be considered when planning future surgery and studies. In particular, comorbid conditions would benefit from higher attendance at follow-ups, including an assessment of sleep apnoea remission. Medium- and long-term follow-up for this cohort would broaden the literature on the topic. Second, while we observed a trend of considerable changes in body composition in our cohort, the one-year values for body composition were elevated by one outlier. This outlier was neither typical of the cohort nor the trend for each individual and, consequently, statistical significance was lacking. Third, the role of the families and social groups of these adolescents should be mentioned. While parental concern about their adolescent 
wards' conditions was noted, preoperative and postoperative psychological evaluations of parents and other family members were not performed. Additionally, the postoperative prevalence of depression could have been assessed among participants as a group. However, as ASMBS paediatric guidelines suggest the involvement of a mental health specialist for children, ${ }^{(29)}$ these adolescents were referred for such care when appropriate.

In conclusion, most morbidly obese adolescents in Singapore who undergo bariatric surgery have obesity-related comorbidities at presentation. Common dietary habits include high-calorie drinks and foods. A family history of obesity is often present and attempts at weight loss via lifestyle modifications are unsuccessful. Our preliminary data showed that bariatric surgical intervention is a safe option for weight loss for obese adolescents in Singapore, with some resolution of associated comorbidities at one year after LSG.

\section{REFERENCES}

1. World Health Organization. Obesity and Overweight: fact sheet [online]. Available at: http://www.who.int/mediacentre/factsheets/fs311/en/. Accessed January 11, 2016.

2. Foo LL, Vijaya K, Sloan RA, Ling A. Obesity prevention and management: Singapore's experience. Obes Rev 2013; 14(Suppl 2):106-13.

3. Kumar S, Zarroug AE, Swain JM. Adolescent bariatric surgery. Abdom Imaging 2012; 37: 725-9.

4. James PT, Leach R, Kalamara E, Shayeghi M. The worldwide obesity epidemic. Obes Res 2001; 9(Suppl 4):228S-33S.

5. Chen LJ, Fox KR, Haase A, Wang JM. Obesity, fitness and health in Taiwanese children and adolescents. Eur J Clin Nutr 2006; 60:1367-75.

6. Bondada S, Jen HC, Deugarte DA. Outcomes of bariatric surgery in adolescents. Curr Opin Pediatr 2011; 23:552-6.

7. Ingelfinger JR. Bariatric surgery in adolescents. N Engl J Med 2011; 365:1365-7.

8. Singapore Ministry of Health. National Health Survey 2010. Available at: http://www.moh.gov.sg/content/moh_web/home/Publications/Reports/2011/ national_health_survey2010.html. Accessed January 31, 2016.

9. Lee YS. Consequences of childhood obesity. Ann Acad Med Singapore 2009; 38:75-7.

10. Must A, Strauss RS. Risks and consequences of childhood and adolescent obesity. Int J Obes Relat Metab Disord 1999; 23(Suppl 2):S2-11.

11. Baur LA, Fitzgerald DA. Recommendations for bariatric surgery in adolescents in Australia and New Zealand. J Paediatr Child Health 2010; 46:704-7.

12. Lee WJ. Surgical treatment of obesity: an Asian perspective. Tzu Chi Med 2007; 19:200-6

13. Moon Han S, Kim WW, Oh JH. Results of laparoscopic sleeve gastrectomy (LSG) at 1 year in morbidly obese Korean patients. Obes Surg 2005; 15:1469-75.

14. Tsai WS, Inge TH, Burd RS. Bariatric surgery in adolescents: recent national trends in use and in-hospital outcome. Arch Pediatr Adolesc Med 2007; 161:217-21.

15. Stefater MA, Jenkins T, Inge TH. Bariatric surgery for adolescents. Pediatr Diabetes 2013; 14:1-12.
16. Inge TH, Zeller MH, Jenkins TM, et al. Perioperative outcomes of adolescents undergoing bariatric surgery: the Teen-Longitudinal Assessment of Bariatric Surgery (Teen-LABS) study. JAMA Pediatr 2014; 168:47-53

17. Pedroso FE, Gander J, Oh PS, Zitsman JL. Laparoscopic vertical sleeve gastrectomy significantly improves short term weight loss as compared to laparoscopic adjustable gastric band placement in morbidly obese adolescent patients. J Pediatr Surg 2015; 50:115-22.

18. Nadler EP, Barefoot LC, Qureshi FG. Early results after laparoscopic sleeve gastrectomy in adolescents with morbid obesity. Surgery 2012; 152:212-7.

19. Ibele AR, Mattar SG. Adolescent bariatric surgery. Surg Clin North Am 2011; 91:1339-51.

20. Park JY, Song D, Kim YJ. Clinical experience of weight loss surgery in morbidly obese Korean adolescents. Yonsei Med J 2014; 55:1366-72.

21. Alqahtani AR, Antonisamy B, Alamri H, Elahmedi M, Zimmerman VA. Laparoscopic sleeve gastrectomy in 108 obese children and adolescents aged 5 to 21 years. Ann Surg 2012; 256:266-73.

22. Al-Sabah SK, Almazeedi SM, Dashti SA, et al. The efficacy of laparoscopic sleeve gastrectomy in treating adolescent obesity. Obes Surg 2015; 25:50-4.

23. Pourcher G, De Filippo G, Ferretti S, et al. Short-term results of single-port sleeve gastrectomy in adolescents with severe obesity. Surg Obes Relat Dis 2015; 11:65-9.

24. Dakour AH, Fawal H, Shaghoury I, Safadi BY. Efficacy and safety of laparoscopic sleeve gastrectomy in Lebanese adolescents. Bariatr Surg Pract Patient Care 2016; 11:78-83.

25. Netzer NC, Stoohs RA, Netzer CM, Clark K, Strohl KP. Using the Berlin questionnaire to identify patients at risk for the sleep apnea syndrome. Ann Intern Med 1999; 131:485-91.

26. Johns MW. A new method for measuring daytime sleepiness: the Epworth sleepiness scale. Sleep 1991; 14:540-5.

27. Kroenke K, Spitzer RL, Williams JB. The PHQ-9: validity of a brief depression severity measure. J Gen Intern Med 2001; 16:606-13.

28. Brethauer SA, Kim J, el Chaar M, et al. Standardized outcomes reporting in metabolic and bariatric surgery. Surg Obes Relat Dis 2015; 11:489-506.

29. Michalsky M, Reichard K, Inge T, et al. ASMBS pediatric committee best practice guidelines. Surg Obes Relat Dis 2012; 8:1-7.

30. Arman GA, Himpens J, Dhaenens J, et al. Long-term (11+years) outcomes in weight, patient satisfaction, comorbidities, and gastroesophageal reflux treatment after laparoscopic sleeve gastrectomy. Surg Obes Relat Dis 2016; 12:1778-86.

31. Pok EH, Lee WJ, Ser KH, et al. Laparoscopic sleeve gastrectomy in Asia: long term outcome and revisional surgery. Asian J Surg 2016; 39:21-8.

32. Mialich MS, Sicchieri JM, Junior AA. Analysis of body composition: a critical review of the use of bioelectrical impedance analysis. Int J Clin Nutr 2014; 2:1-10.

33. Friedrich AE, Damms-Machado A, Meile T, et al. Laparoscopic sleeve gastrectomy compared to a multidisciplinary weight loss program for obesity-effects on body composition and protein status. Obes Surg 2013; 23:1957-65.

34. Fernstrom MH, Courcoulas AP. Bariatric surgery for the severely obese adolescent. Aesthet Surg J 2008; 28:331-4.

35. Rosenthal RJ, Diaz AA, Arvidsson D, et al. International Sleeve Gastrectomy Expert Panel Consensus Statement: best practice guidelines based on experience of $>12,000$ cases. Surg Obes Relat Dis 2012; 8:8-19.

36. Kraus C, DeUgarte D, Feinfeld LE. Bariatric surgery in adolescents. Infant Child Adolesc Nutr 2010; 2:294-8

37. Herget S, Rudolph A, Hilbert A, Blüher S. Psychosocial status and mental health in adolescents before and after bariatric surgery. A systematic literature review. Obes Facts 2014; 7:233-45. 